\title{
Video- und protokollbasierte Reflexionen eigener praktischer Unterrichtserfahrungen im Lehramtsstudium
}

\author{
Denise Kücholl (D) - Rebecca Lazarides
}

Eingegangen: 22. April 2020 / Überarbeitet: 21. Januar 2021 / Angenommen: 28. April 2021 / Online publiziert: 16. Juni 2021

(C) Der/die Autor(en) 2021

Zusammenfassung Die Reflexion praktischer Unterrichtserfahrungen ermöglicht es Lehramtsstudierenden theoretische Wissensaspekte und praxisrelevante Erkenntnisse miteinander zu verknüpfen. Die vorliegende Studie untersucht die Bedeutung des Mediums der Reflexion (eigenes Video vs. fremdes Video vs. Protokoll) sowie der Art der reflektierten Unterrichtssituation (positiv vs. herausfordernd) für die Reflexionsprozesse Lehramtsstudierender. Darüber hinaus wird untersucht, auf welchen Ebenen (Basal-, Sicht- und Tiefenstrukturen) das Unterrichtsgeschehen reflektiert wird. Datengrundlage der quasi-experimentellen Studie sind Unterrichtsreflexionen von 55 Lehramtsstudierenden, die inhaltsanalytisch ausgewertet wurden. Die Ergebnisse zeigen, dass Lehramtsstudierende die Tiefenstrukturen des Unterrichts reflektieren, wenn die Reflexion von Situationen, die als positiv erlebt wurden, anhand fremder Unterrichtsvideos geschieht. Bei der Reflexion von herausfordernden Unterrichtssituationen werden Tiefenstrukturen sowohl anhand von fremden Videos als auch anhand von Protokollen reflektiert. Die Implikationen der Ergebnisse für die Lehrkräftebildung werden im Hinblick auf die Reflexion von Unterrichtspraxis diskutiert.

Schlüsselwörter Reflexionsprozesse · Lehrkräftebildung · Unterrichtspraxis · Videobasierte Reflexion

\footnotetext{
Dr. Denise Kücholl $(\bowtie)$

Department für Erziehungswissenschaft, Universität Potsdam, Karl-Liebknecht-Straße 24-25, 14476 Potsdam, Deutschland

E-Mail: denise_kuecholl@web.de

Prof. Dr. Rebecca Lazarides

Professur für Schulpädagogik/Empirische Unterrichtsforschung, Universität Potsdam, Karl-Liebknecht-Str. 24-25, 14476 Potsdam, Deutschland

E-Mail: rebecca.lazarides@uni-potsdam.de
} 


\title{
Video and text-based reflections of practical experiences at preservice teachers
}

\begin{abstract}
The reflection of practical experiences of teaching enables preservice teachers to combine aspects of theoretical and practical knowledge. Therefore, the current study investigates the meaning of the medium-that means video compared to text-based reflections - and the type of situations - that are positive or difficult situations of teaching - and relations to preservice teachers' reflective processes. Also, levels of reflections are investigated. In our study, we examine reflections of 55 preservice teachers using a quasi-experimental design. Qualitative content analysis shows that preservice teachers reflect deep levels of teaching while using videos of peers in situations that they evaluated as positive experiences in school. However, when rating situations that were evaluated as challenging preservice teachers reflected deep levels when using videos of peers as well as text-based reflections. Implications of the study for teacher training in reflection are discussed.
\end{abstract}

Keywords Practical experiences $\cdot$ Reflection $\cdot$ Teacher training $\cdot$ Video-based reflection

\section{Einleitung}

Praxisphasen im Lehramtsstudium sind bedeutsam für den Professionalisierungsprozess - insbesondere aus Sicht der Lehramtsstudierenden selbst, denn sie können entscheidend dazu beitragen, professionelle Unterrichtskompetenzen zu erwerben (vgl. Drahmann et al. 2018; Gröschner und Hascher 2019). Obwohl erste Praxisphasen häufig mit vielfältigen neuen Anforderungssituationen verbunden sind (KellerSchneider 2016), wünschen sich Lehramtsstudierende explizit, eigene Fähigkeiten in der Berufspraxis erproben zu können (Hascher 2014). Die Reflexion, d. h. das gezielte Nachdenken und Nachvollziehen der schulpraktischen Unterrichtserfahrungen, ist als weiteres wichtiges Element des Professionalisierungsprozesses anzusehen (Gläser-Zikuda et al. 2019; Wyss 2018). Reflexionsprozesse im Lehramtsstudium ermöglichen die Verknüpfung theoretischen Professionswissens mit erfahrungsbasiertem berufspraktischem Wissen (Korthagen 2010). Empirische Studien, die Reflexionsprozesse in schulpraktischen Lehrveranstaltungen untersuchten, befassten sich bislang insbesondere mit der Frage, welche Bedeutung das zugrundeliegende Medium für die Reflexion innehat. Zum Beispiel wird untersucht, wie verschiedene Unterrichtssituationen anhand eines eigenen vs. eines fremden Videos bzw. anhand eines Protokolls reflektiert werden (vgl. Kleinknecht und Gröschner 2016). Eine offene Frage ist bislang, inwiefern es abgesehen vom genutzten Medium für die Schritte im Reflexionsprozess bedeutend ist, ob eine als positiv wahrgenommene bzw. eine als herausfordernd wahrgenommene Situation reflektiert wird (Hamel und ViauGuay 2019). Des Weiteren ist gegenwärtig wenig bekannt zu den Ebenen, auf denen Lehramtsstudierende das Unterrichtsgeschehen reflektieren, d.h. inwieweit basale bzw. Sicht- und Tiefenstrukturen reflektiert werden. Ungeklärt ist zudem, inwieweit die Art der reflektierten Situation (positiv oder herausfordernd) bedeutsam ist für 
die Ebene des Reflektierens (z.B. basale Beobachtungen vs. Thematisierung der Prozessqualität). Des Weiteren ist bislang nur wenig bekannt dazu, inwieweit die Ebenen der Reflexion auch mit der Art des im Reflexionsprozess genutzten Mediums (z. B. Video oder Protokoll) zusammenhängen (Rosaen et al. 2010). Die vorliegende Studie widmet sich diesen Forschungsdesideraten.

\section{Theoretischer Hintergrund}

\subsection{Reflexion als Kompetenz im Lehrberuf}

Die Fähigkeit Unterrichtserfahrungen zu reflektieren ist als zentrales Element professioneller Kompetenz von angehenden Lehrkräften einzuschätzen (Altrichter 2000). Angelehnt an Wyss (2018) kann Reflexion definiert werden als ,ein gezieltes, aufmerksames Nachdenken über bestimmte Handlungen, Gedanken und Geschehnisse“ (Wyss 2018, S. 19). Das Unterrichtsgeschehen wird dementsprechend systematisch und anhand von Kriterien ergründet. Reflexion kann als eine spezifische Form des bewussten Nachdenkens aufgefasst werden, mithilfe dessen ein bestimmtes Ziel verfolgt wird (Wyss 2013). In ihrer Forschung knüpfte Wyss (2013) insbesondere an die Arbeiten von Schön (1983) an, der in „The Reflective Practioner“ die Bedeutung von Reflexionen im Lehrberuf herausstellte und dabei die enge Verknüpfung zwischen der Reflexion, dem individuellen Handeln im Unterricht und der persönlichen Kompetenzentwicklung betonte. Reflexionen können dabei unterstützen, Prozesse des Handelns und Denkens zu ergründen, zu erklären und zu verbessern (Schön 1983). Als wesentlich gilt dabei, Geschehnisse aus einer erweiterten Perspektive zu betrachten und über alternatives Verhalten nachzudenken. Zudem können Reflexionen ermöglichen, die subjektive Wahrnehmung und Interpretation zu hinterfragen und anzupassen, so dass Veränderungsprozesse angeregt werden können (GläserZikuda et al. 2019). Die Reflexion von praktischen Unterrichtserfahrungen kann darüber hinaus entscheidend dazu beitragen, dass sich Lehramtsstudierende hinsichtlich ihrer Expertise entwickeln (Berliner 2004). Das bewusste Nachvollziehen von Erfahrungswissen in Verbindung mit der Vertiefung von kontextspezifischen Kenntnissen im Unterrichten sowie die Entwicklung hilfreicher Problemlösestrategien können dabei als wesentlich für die Entwicklung von Expertise betrachtet werden (Gruber und Hascher 2011). Reusser und Wyss (2000) betonen zudem, dass die Fähigkeit schulpraktische Erfahrungen zu reflektieren als „Gradmesser der professionellen Qualifikation“ anzusehen ist (Reusser und Wyss 2000, S. 9). In der Lehrerinnen- und Lehrerbildung wird der Fähigkeit zu reflektieren international seit geraumer Zeit ein hohes Potenzial zugesprochen (Wyss 2018). Reflexivität wird vielfach als Schlüsselkompetenz von Lehrkräften bezeichnet, wobei sie einen wichtigen Stellenwert eingenommen hat und verbindliche Vorgaben dazu formuliert wurden (vgl. Clarà 2015; Combe und Kolbe 2008). Als Kernaufgabe von Lehrkräften wird von der Kultusministerkonferenz ,die gezielte und nach wissenschaftlichen Erkenntnissen gestaltete Planung, Organisation und Reflexion von Lehr- und Lernprozessen sowie ihre individuelle Bewertung und systemische Evaluation“ definiert (Kultusministerkonferenz 2019, S. 3). Theoretische Bezüge lassen sich zudem zwischen 
der Forschung zu Reflexion im Lehrberuf und etablierten Modellen professioneller Handlungskompetenzen von Lehrkräften herstellen (Baumert und Kunter 2006). Die Fähigkeit zur Reflexion des Unterrichtsgeschehens könnte hier dem Bereich selbstregulativer Fähigkeiten zugeordnet werden, der die Steuerung und die Regulation des eigenen Handelns sowie den verantwortungsbewussten Umgang mit Ressourcen umfasst. Konkreter kann die Fähigkeit zu reflektieren als Fähigkeit eigenes Handeln zu regulieren - also zu planen und ggf. anzupassen - verstanden werden und damit metakognitiven Komponenten selbstregulativer Fähigkeiten zugeordnet werden (Landmann et al. 2015).

\subsection{Reflexion als Prozess}

Das Reflektieren kann als Prozess beschrieben werden, der unterschiedliche Schritte umfasst und unter Bezugnahme von individuellen Wertvorstellungen der Lehramtsstudierenden, ihren Erfahrungen und ihren subjektiven Überzeugungen eine erweiterte Perspektive ermöglicht, die Aspekte des Unterrichtsgeschehens betrifft (Wyss 2018). Reflexionen, die einem Prozess der Distanzierung unterliegen, können im Sinne eines rückblickenden Deutens und Evaluierens erlebter Unterrichtssituationen verstanden werden (vgl. Combe und Kolbe 2008). Motiviert durch die Wahrnehmung eines Moments der Verunsicherung findet laut Dewey (1933) das Suchen nach möglichen Erklärungen statt, die möglichst zur Auflösung aufgekommener Zweifel führen sollen. Anknüpfend an die Arbeiten von Dewey (1933) beschreibt Schön (1983) sechs Phasen des Reflexionsprozesses: Nach der Wahrnehmung einer Diskrepanz zwischen Erwartung und Realität entsteht Verunsicherung (Schritt 1), das Problem wird benannt (Schritt 2), definiert und auf vergleichbare Situationen in der Vergangenheit bezogen (Schritt 3). Schließlich wird begründet, wodurch sich das Problem ergeben hat (Schritt 4) und es werden Konsequenzen abgeleitet (Schritt 5). Werden diese Konsequenzen vom Handelnden als günstig wahrgenommen, erfolgt die Entwicklung möglicher alternativer Verhaltensstrategien (Schritt 6) (Schön 1983). Hatton und Smith (1995) gehen bezugnehmend auf die Arbeiten von Schön (1983) von vier Formen des Reflektierens aus: descriptive writing (Beschreibung), descriptive reflection (Entwicklung von Begründungen), dialogic reflection (Abwägen von Deutungen und Formulierung von Alternativen) und critical reflection (Einbeziehung des historischen und gesellschaftspolitischen Kontextes). Daran anknüpfend und in Bezug auf die Forschung zur professionellen Unterrichtswahrnehmung geben Kleinknecht und Gröschner (2016) in ihren Forschungsarbeiten drei Stufen als Struktur für die Reflexion vor: Im ersten Schritt werden die Lehramtsstudierenden gebeten, eine Situation zu beschreiben, im zweiten Schritt soll das eigene Handeln begründet und bewertet werden und im dritten Schritt gilt es, alternative Handlungsstrategien zu formulieren.

Das Konzept der Reflexion weist theoretische Parallelen zum Konzept der professionellen Unterrichtswahrnehmung auf. Das Erkennen relevanter Situationen, auch bezeichnet als „noticing“ bzw. ,,selective attention“ kann als wesentliche Voraussetzung für das Beschreiben, der ersten Form des Reflektierens, angesehen werden (vgl. Hatton und Smith 1995; Kleinknecht und Gröschner 2016). Weiterhin umfasst das 
Konzept der professionellen Unterrichtswahrnehmung das wissensbasierte Begründen der Unterrichtssituationen, auch bezeichnet als ,reasoning“ (van Es und Sherin 2002). Hierbei ist eine deutliche Parallele zur zweiten Form ,descriptive reflection“, d.h. der Begründung von Unterrichtshandeln, zu erkennen. Als wesentliches Unterscheidungsmerkmal der beiden Konzepte ist anzusehen, dass das Konzept der Reflexion das Abwägen im Sinne eines Prüfens der gedeuteten Begründungen sowie das Formulieren von Alternativen als dritten Schritt des Reflektierens konzipiert (vgl. Hatton und Smith 1995), während dieser Schritt im Konzept der professionellen Unterrichtswahrnehmung nicht beschrieben wird.

Lernrelevante Unterrichtssituationen zu beschreiben, sie analysierend zu begründen und zu bewerten sowie entsprechende Handlungsalternativen abzuleiten - die Reflexion des Unterrichts also - ist eine zentrale Voraussetzung für professionelles Handeln im Unterricht (Blömeke et al. 2014). Die Fähigkeit, die einzelnen Reflexionsschritte zu vollziehen, hängt unter anderem von der Berufserfahrung ab. Empirische Arbeiten zu professioneller Unterrichtswahrnehmung konnten beispielsweise zeigen, dass Lehramtsstudierende zwar in der Lage sind, einzelne Unterrichtssituationen detailliert $\mathrm{zu}$ beschreiben, dass sie jedoch im Vergleich $\mathrm{zu}$ erfahrenen Lehrkräften weniger gut in der Lage sind, Unterrichtsstrukturen in ihrer gesamten Komplexität wahrzunehmen und den Gesamtkontext des Geschehens zu erfassen (Seidel und Prenzel 2007). Zudem gelingt es ihnen weniger gut, die Geschehnisse präzise zu deuten und zu erläutern (Klassen und Durksen 2014). Darüber hinaus fällt es Lehramtsstudierenden schwer, mögliche alternative Handlungsformen zu benennen und zu erklären (Schäfer und Seidel 2015). Da die vorliegende Arbeit sich mit der Frage nach Reflexionsprozessen im Lehramtsstudium befasst, beziehen wir uns in unserer Studie auf das angeleitete, strukturierte Vorgehen nach Kleinknecht und Gröschner (2016).

\subsection{Bedeutung von Situationen und Ebenen des professionellen Handelns für Reflexionsprozesse}

Für Reflexionsprozesse im Lehramtsstudium ist neben der Art und Weise wie Lehramtsstudierende reflektieren, d. h. welche Schritte oder Phasen des Reflexionsprozesses durchlaufen werden, gleichzeitig von Bedeutung, welche Art von Unterrichtssituationen reflektiert wird. Obwohl Reflexionen primär durch Momente der Verunsicherung und des Zweifelns ausgelöst werden (Dewey 1933), können auch als gelungen wahrgenommene Situationen Anlass für Reflexionen bieten. Das Wahrnehmen und Reflektieren von erfolgreichem Handeln kann die Entwicklung professioneller Unterrichtskompetenzen begünstigen (Heemsoth 2019). Die Auseinandersetzung mit eigenen Erfolgserfahrungen kann zur Entwicklung kognitiver, verhaltensbezogener und selbstregulativer Fähigkeiten beitragen, die zudem sukzessive den effektiven Umgang mit als herausfordernd wahrgenommenen Situationen begünstigen (,,mastery experiences“; Bandura 1997). Situationen hingegen, die Lehramtsstudierende als herausfordernd wahrnehmen, sind häufig in besonderem Maße mit Irritationen oder Abwehr verbunden (Seidel et al. 2011). Lehrkonzepte, die neben gelungenem Handeln auch auf das Reflektieren ungünstigen Handelns setzen und problemorientiertes Lernen fokussieren, haben sich vielfach als lernwirksam erwiesen, da sie komplexe 
kognitive Prozesse begünstigen (Heemsoth 2019; Stark et al. 2010). Anknüpfend an die Forschung zur Arbeit mit Problemsituationen kann daher angenommen werden, dass besonders die Auseinandersetzung mit als herausfordernd wahrgenommenen Situationen zu komplexen Reflexionsprozessen führt, die sich beispielsweise im Entwickeln von weiterführenden Handlungsalternativen zeigen (Oser und Spychinger 2005).

Für die Reflexion des professionellen Handelns ist es ebenfalls bedeutend, dass Lehramtsstudierende und Lehrkräfte dazu in der Lage sind, sowohl die Sicht- als auch die Tiefenstrukturen des Unterrichts zu reflektieren (Drechsel und Schindler 2019). Die Unterscheidung von Sicht- und Tiefenstrukturen ist in der Unterrichtsforschung seit Langem etabliert (Aebli 1961; Oser und Baeriswyl 2001; Reusser 2016). Insbesondere Aebli (1961) legte mit seinem Konzept kognitionspsychologischer Didaktik den Grundstein dafür, spezifische Lern- und Verstehensprozesse der Lernenden zu betrachten. Sichtstrukturen - auch als Oberflächenstrukturen bezeichnet werden unmittelbar durch das Betrachten der Unterrichtssituation wahrgenommen und können beispielsweise Organisationsformen (z. B. Frontalunterricht), Methoden des Unterrichts (z. B. Projektorientierter Unterricht) oder Sozialformen (z. B. Einzel-, Partner- oder Gruppenarbeit) betreffen (Kunter und Ewald 2016). Tiefenstrukturen des Unterrichts hingegen beschreiben, auf welche Art und Weise sich die Lernenden mit den Lerngegenständen auseinandersetzen und betreffen die psychologischdidaktischen Prozessqualitäten. Dazu zählen spezifische Prozesse, die der Qualität des Lern- und Arbeitsgeschehens zugrunde liegen wie beispielsweise wertschätzende Interaktionen zwischen den Lehrkräften und den Lernenden oder der Grad der kognitiven Anregung, der durch den Einsatz bestimmter Methoden initiiert wird (Oser und Baeriswyl 2001). Die Tiefenstrukturen des Unterrichts zu reflektieren, erfordert das Geschehen zuweilen stärker zu interpretieren (Drechsel und Schindler 2019). Während es zahlreiche Arbeiten dazu gibt, inwiefern Lehrkräfte die Sichtund Tiefenstrukturen des Unterrichts beurteilen und inwieweit ihre Beurteilungen mit denen von Lernenden vergleichbar sind (Wagner et al. 2016), ist bislang ungeklärt, inwiefern Lehramtsstudierende oder Lehrkräfte ihren Unterricht auch auf den verschiedenen Ebenen des Unterrichts reflektieren.

\subsection{Bedeutung des genutzten Mediums für Reflexionsprozesse im Lehramtsstudium}

Forschungsarbeiten verweisen darauf, dass videografierte Unterrichtssequenzen angehenden Lehrkräften einen spezifischen Zugang zu eigenen und fremden praktischen Erfahrungen ermöglichen können (Wyss 2013). Videografierte Sequenzen können es Lehramtsstudierenden aufgrund ihrer Realitätsnähe, ihrer Informationsfülle und ihrer Anschaulichkeit ermöglichen, sich gezielt der Komplexität von Unterrichtssituationen zu widmen und bieten die Möglichkeit hoher kognitiver und emotionaler Aktivierung während der Reflexion (Krammer und Reusser 2005). Dabei können komplexe Unterrichtssituationen im Ganzen abgebildet werden, sodass das Handeln aus unterschiedlichen Perspektiven wahrgenommen und interpretiert sowie alternative Handlungsformen entwickelt werden können (Häusler et al. 2019). Insbesondere Studierende mit wenigen praktischen Unterrichtserfahrungen scheinen von 
einer angeleiteten und strukturierten Reflexion ihres praktischen Handelns anhand von Videos zu profitieren. Anscheinend begünstigt dabei insbesondere die Reflexion fremden videografierten Unterrichtshandelns den Reflexionsprozess (Seidel et al. 2013). Es zeigte sich, dass angehende Lehrkräfte, die Unterricht anhand eigener Videos reflektieren, das eigene Handeln im Zuge der Selbstreflexion im Vergleich zur Reflexion fremden Verhaltens weniger umfassend beschreiben (Kleinknecht und Gröschner 2016) und seltener Begründungen für ihr Verhalten vornehmen (Seidel et al. 2011). Lehramtsstudierende, die Unterricht anhand von fremden Videos reflektieren, bewerten das Verhalten im Vergleich zu Lehramtsstudierenden, die anhand eigener Videos reflektieren, häufig besonders kritisch (Seidel et al. 2011), entwickeln jedoch auch häufiger Handlungsalternativen. Studien zeigen des Weiteren, dass Lehramtsstudierende bei Reflexionen anhand von Protokollen im Vergleich zu eigenen Videos die Unterrichtssituationen tiefgründiger beschreiben, bewerten und begründen und vermehrt Handlungsalternativen entwickeln (Kleinknecht und Gröschner 2016; Schneider et al. 2016). Diskutiert wird in diesem Kontext, inwieweit Reflexionen anhand von Videografie aufgrund der hohen Authentizität und Komplexität insbesondere Lehramtsstudierende mit wenigen Unterrichtserfahrungen kognitiv überfrachten (Kramer et al. 2017; Syring et al. 2015). Inwieweit das verwendete Medium im Zusammenhang mit den einzelnen Schritten während des Reflexionsprozesses steht, ist laut derzeitiger empirischer Studienlage weitgehend offen.

\section{Die vorliegende Studie}

Reflexionsprozesse sind für Lehramtsstudierende in Praxisphasen sowie für Lehrkräfte in der beruflichen Arbeit bedeutsam für die Entwicklung professioneller Kompetenzen, für das Wohlbefinden und für den Umgang mit belastenden Unterrichtssituationen (Gläser-Zikuda et al. 2019). Die aktuelle Befundlage zeigt, dass Lehramtsstudierende - sowohl anhand von videografiertem Unterricht als auch anhand von Protokollen - sämtlichen Schritten im Reflexionsprozess nachgehen, wenn sie zu einer strukturierten Reflexion angeleitet werden (Kleinknecht und Schneider 2013). Bislang existieren jedoch kaum Befunde dazu, inwieweit die Art der Situation und das bei der Reflexion genutzte Medium den Prozess der Reflexion beeinflussen. Zudem ist nur wenig dazu bekannt, auf welchen Ebenen Lehramtsstudierende das Unterrichtsgeschehen reflektieren (Drechsel und Schindler 2019). Diese Forschungsdesiderata greift die vorliegende Untersuchung auf. Dabei untersuchen wir folgende Forschungsfragen:

Forschungsfrage 1 Welche Bedeutung hat die Art der reflektierten Unterrichtssituation, also ob eine positive oder herausfordernde Situation reflektiert wird, für den Reflexionsprozess?

Anknüpfend an die Ausführungen von Dewey (1933) nehmen wir an, dass bei der Reflexion von als herausfordernd wahrgenommenen Situationen komplexere Reflexionsprozesse angeregt werden, da nach dem Aufkommen von Zweifeln Ursachen ergründet werden und möglichst die Auflösung aufgekommener Zweifel angestrebt 
wird. Darüber hinaus lässt sich unsere Annahme anknüpfen an die dargestellte Forschung zum problembasierten Lernen (Oser und Spychinger 2005; Stark et al. 2010). Es konnte gezeigt werden, dass Lehrkonzepte, die sich auf die Reflexion schwieriger Situationen fokussieren, komplexe Reflexionsprozesse begünstigen können (Heemsoth 2019). Zudem kann entsprechend der Theorie zu Selbstwirksamkeitserwartungen nach Bandura (1997) angenommen werden, dass die Überwindung erlebter Irritationen innerlich als Erfolgserlebnis (,Mastery-Experience“) bewertet wird und dazu beiträgt, dass Personen sich zukünftig ähnlich herausfordernden Situationen zuversichtlich widmen.

Forschungsfrage 2 Welche Bedeutung hat das verwendete Medium - d. h. die Verwendung eines Videos bzw. eines Protokolls, das im eigenen Unterricht entstanden ist - für den Reflexionsprozess?

Bezugnehmend auf bisherige Forschungsarbeiten (Kleinknecht und Gröschner 2016) wird angenommen, dass besonders die Reflexion anhand fremder Videos bedeutend für die Schritte im Reflexionsprozess ist, so dass komplexe Reflexionsschritte vollzogen werden. Für die Reflexion anhand von Protokollen wird angenommen, dass diese im Vergleich zur Reflexion anhand eigener Videos eher komplexere Formen der Reflexion begünstigen.

Forschungsfrage 3 Welche Bedeutung hat zum einen die Art der Situation (positiv vs. herausfordernd) und zum anderen das Medium, das der Reflexion zugrunde liegt (eigenes Video, Protokoll, fremdes Video) für die Ebenen der Reflexion (Basal-, Sicht- bzw. Tiefenstrukturen), wenn komplexe Reflexionsschritte wie das Formulieren von Alternativen vorgenommen werden?

Die Forschungsfrage bezieht sich insbesondere auf den komplexen Reflexionsschritt des Entwickelns von Alternativen, da hierbei besonders komplexe kognitive Prozesse notwendig sind. Wissen um Bedingungsfaktoren, die derartige Prozesse begünstigen können, ist von hoher Relevanz für ein gezieltes Nachdenken über Unterrichtshandeln. Es wird angenommen, dass als herausfordernd wahrgenommene Situationen dazu anregen und zudem die Reflexion von Sicht- und Tiefenstrukturen begünstigen (Heemsoth 2019). Aufbauend auf den Ausführungen von Oser und Spychinger (2005) und Heemsoth und Kleickmann (2018) nehmen wir an, dass insbesondere die Auseinandersetzung mit Herausforderungen und Schwierigkeiten tiefgründiges Reflektieren begünstigt, da Lehramtsstudierende Irritationen ergründen und überwinden wollen.

Ebenfalls nehmen wir an, dass Reflexionen anhand von Videografie eher mit Reflexionen der Tiefenstrukturen einhergehen, da ein spezifischerer und umfassenderer Zugang zum Geschehen ermöglicht wird (Häusler et al. 2019). Es wird davon ausgegangen, dass Videografie eine komplexe Reflexion - jenseits sichtbarer Methoden und Sozialformen (Sichtstrukturen) - ermöglicht, da Geschehnisse und Prozesse aus unterschiedlichen Perspektiven betrachtet werden können (Krammer und Reusser 2005). Gleichzeitig konnte in Erhebungen gezeigt werden, dass Reflexionen anhand von Videos kognitiv überfordern können (Syring et al. 2015). Protokolle könnten Lehramtsstudierenden ermöglichen, das Geschehen mit Abstand zu reflektieren, so dass eine Entlastung von Handlungsdruck stattfinden kann (Kramer et al. 2017). 


\section{Methode}

\subsection{Interventionsdesign}

Die Daten der vorliegenden Studie wurden im Rahmen einer Intervention zur Förderung der Reflexionskompetenz und Selbstwirksamkeitseinschätzungen erfasst (Lazarides 2018). Die Intervention ist im Rahmen von schulnetzwerkintegrierten Praxisseminaren im Bachelorstudium im Studienbereich für Bildungswissenschaften für das Lehramt für die Sekundarstufe I und II (allgemeinbildende Fächer) implementiert (vgl.: Lazarides 2018). Die Seminare sind inhaltlich gleich und standardisiert in ihrer Durchführung. Im ersten Schritt wird theoretisches Wissen zum Thema „Motivierendes Unterrichten“ vermittelt. Dazu setzen sich die Lehramtsstudierenden verschiedener Fachrichtungen zunächst mit psychologischen Motivationstheorien auseinander. Im zweiten Schritt werden die vermittelten theoretischen Kenntnisse praktisch angewandt. Dazu werden die Lehramtsstudierenden den teilnehmenden Lehrkräften im Netzwerk entsprechend ihres Faches zugeordnet. Nach einer Hospitation von mindestens 90 min entwickeln die Lehramtsstudierenden in Absprache mit den Lehrkräften fachbezogene Unterrichtsentwürfe. Nach dem Erhalt systematischen Feedbacks durch die Dozierenden sowie durch die Lehrkräfte und durch die Mitstudierenden werden die Unterrichtsentwürfe im Fachunterricht der jeweiligen Netzwerkschulen in der Praxis umgesetzt. Insofern in der teilnehmenden Schule die Erlaubnis zur Videografie des Unterrichts erteilt wurde, wird der Unterricht aufgezeichnet. Ist dies nicht möglich, wird der Unterricht mithilfe eines standardisierten, narrativen Protokolls schriftlich dokumentiert. Im dritten Schritt werden die Teilnehmenden mit Hilfe eines schriftlichen Reflexionsauftrags dazu angeleitet, Reflexionen von jeweils ca. einer DIN-A4-Seite zu verfassen. Zum einen soll eine Reflexion zu einer Situation verfasst werden, in der die Aspekte motivierenden Unterrichtens gelungen umgesetzt werden konnten, d.h. eine als positiv wahrgenommene Situation. Zum anderen werden die Studierenden aufgefordert, eine Situation auszuwählen, die sie als problematisch hinsichtlich der Umsetzung der Aspekte motivierenden Unterrichtens einschätzen, d.h. einer als herausfordernd wahrgenommenen Situation. Die Studierenden werden für die Reflexionsphase drei Gruppen zugeteilt. Lehramtsstudierende, deren Unterricht aufgezeichnet werden konnte, reflektieren entweder anhand ihres eigenen Videos (IG 1) oder anhand des Videos einer/eines Mitstudierenden (IG 3). Insofern die Aufzeichnung des Unterrichts nicht ermöglicht werden konnte, wird anhand des schriftlich erstellten Protokolls reflektiert (IG 2). Lehramtsstudierende der IG 1 und IG 3 nutzen Videosequenzen einer Gesamtdauer von jeweils ca. fünf Minuten, wobei sie selbst entscheiden können, welcher Sequenz sie sich widmen. Das schriftliche Protokoll umfasst in der Regel zwischen einer halben und einer ganzen DIN-A4-Seite. Zu ihrer Reflexion der als herausfordernd wahrgenommenen Situation erhalten die Lehramtsstudierenden ein Peer-Feedback. Insgesamt liegen für die vorliegende Studie $N=50$ schriftliche Reflexionen zu positiven Situationen und $N=55$ Reflexionen zu herausfordernden Situationen vor. 


\subsection{Stichprobe}

Die Datengrundlage der hier vorgestellten quasi-experimentellen Studie umfasst 55 Lehramtsstudierende (58,2\% weiblich; 36,4\% männlich; 5,5\% keine Angabe). Besonders häufig vertreten waren Studierende der Fächer Deutsch (14,7\%), Lebensgestaltung-Ethik-Religionskunde $(12,1 \%)$ und Sport $(9,5 \%)$. Die Studierenden befanden sich zum großen Teil $(38,5 \%)$ im 3. oder 4 . Fachsemester $(32,1 \%$ im 5. oder 6. Fachsemester; 5,8\% im 1. bis 2. Fachsemester; 1,1\% im $\geq 9$. Fachsemester und $1 \%$ im 7. bis 8. Fachsemester). Da das Seminar im Studienverlaufsplan am Ende des Bachelorstudiengangs verortet ist, verfügen die Studierenden über erste schulpraktische Erfahrungen, da sie im Rahmen der schulpraktischen Studien sowohl bereits das Orientierungspraktikum, das Praktikum in einem pädagogischpsychologischen Handlungsfeld sowie fachdidaktische Tagespraktika absolviert haben. Die Studierenden waren zum Zeitpunkt der Untersuchung zwischen 19 und 34 Jahren alt $(M=22,99, S D=2,79)$. Die einbezogenen Daten wurden über zwei Semester in jeweils drei Seminargruppen erhoben. Die Studierenden wurden folgenden Gruppen zugeteilt: Gruppe „eigenes Video“ (IG 1; $n=16$ Alter: $M=22,15$ Jahre, $S D=1,87 ; 43,75 \%$ weiblich; 43,75\% männlich), Gruppe „Protokoll“ (IG 2; $n=17$; Alter: $M=22,84, S D=2,38 ; 81,82 \%$ weiblich; $13,64 \%$ männlich), Gruppe ,fremdes Video" (IG 3; $n=22$; Alter: $M=24,00, S D=3,80 ; 41,17 \%$ weiblich; $52,94 \%$ männlich). Die Studierenden in den einzelnen Gruppen unterschieden sich nicht hinsichtlich der Semesterzahl, $F(2 ; 34,54)=3,06, p=0,062$, der Anzahl der Fachsemester, $F(2 ; 5,47)=0,19, p=0,827$ oder der pädagogischen Vorerfahrungen (in Monaten $), F(2 ; 7717,71)=1,25, p=0,300$. Signifikante Unterschiede in der Geschlechterverteilung existierten insofern, als dass in der Gruppe „Protokoll“ (IG 2) mehr Frauen als Männer teilnahmen, $\chi^{2}(2)=8,07, p=0,018$.

\subsection{Entwicklung des Kategoriensystems und Auswertung der Reflexionen}

Zur Auswertung der Reflexionen wurde angelehnt an die theoretischen Arbeiten nach Kleinknecht und Gröschner (2016) ein Kategoriensystem entwickelt, mithilfe dessen die Reflexionsschritte Beschreiben, Begründen und Bewerten sowie Alternativen entwickeln ausgewertet werden können. Orientierung bei der Entwicklung des Kategoriensystems bot das von Lohse-Bossenz und Krauskopf (2017) entwickelte Kategoriensystem zur Bewertung schriftlicher Reflexionen. In diesem Kategoriensystem werden die drei Reflexionsschritte Beschreiben, Begründen und Bewerten sowie Alternativen entwickeln jeweils als Oberkategorie benannt und zugleich definiert. Die entsprechenden Oberkategorien werden auch als Grundlage für die vorliegende Arbeit genutzt. In der vorliegenden Arbeit wurde das bestehende Kategoriensystem nach Lohse-Bossenz und Krauskopf (2017) erweitert, indem die Betrachtungsebenen von Unterricht als Elemente der Kategorien zusätzlich ergänzt wurden, um auswerten zu können, auf welcher Ebene das Unterrichtsgeschehen reflektiert wurde. Eng orientiert an den theoretischen Arbeiten von Aebli (1961), Oser und Baeriswyl (2001) sowie Reusser (2016) wurden die Unterkategorien Sichtstrukturen sowie Tiefenstrukturen benannt und definiert. Ebenfalls wurden zu den beiden Unterkategorien Kodierregeln formuliert (vgl. Tab. 1). 
Tab. 1 Reflexionsschritt 3: Alternativen entwickeln mit Ebenen der Reflexionen und Definitionen, Ankerbeispielen und Kodierregeln; Subkategorie: Alternativen zu Handlungen unter Beteiligung der/des Lehramtsstudierenden

\begin{tabular}{|c|c|c|c|}
\hline & Definition & Ankerbeispiel & Kodierregel \\
\hline $\begin{array}{l}\text { Basal- } \\
\text { strukturen }\end{array}$ & $\begin{array}{l}\text { Formulierung von } \\
\text { Alternativen zu } \\
\text { den Handlungen } \\
\text { der/des Lehramts- } \\
\text { studierenden ohne } \\
\text { direkten und kon- } \\
\text { kreten Bezug auf } \\
\text { Organisationsfor- } \\
\text { men, Methoden } \\
\text { und Sozialformen }\end{array}$ & $\begin{array}{l}\text { "Die Lehrkraft hätte in dieser } \\
\text { Situation standhaft bleiben } \\
\text { müssen." }\end{array}$ & $\begin{array}{l}\text { + wird vergeben, wenn Alter- } \\
\text { nativen zu den Handlungen } \\
\text { der Lehrkraft formuliert wer- } \\
\text { den, die sich auf offensichtlich } \\
\text { erkennbare Aspekte des Unter- } \\
\text { richts und der Handlungen der } \\
\text { Lehrkraft beziehen, die aber } \\
\text { keinen Bezug zu den Organi- } \\
\text { sationsformen, Methoden und } \\
\text { Sozialformen haben und auch } \\
\text { keinen konkreten Bezug zu } \\
\text { Interaktionsprozessen }\end{array}$ \\
\hline $\begin{array}{l}\text { Sicht- } \\
\text { strukturen }\end{array}$ & $\begin{array}{l}\text { Formulierung von } \\
\text { Alternativen zu } \\
\text { den Handlungen } \\
\text { der/des Lehramts- } \\
\text { studierenden mit } \\
\text { Bezug auf Orga- } \\
\text { nisationsformen, } \\
\text { Methoden und } \\
\text { Sozialformen }\end{array}$ & $\begin{array}{l}\text { „Die Lehrkraft hätte trotz } \\
\text { der Beschwerden vonseiten } \\
\text { der Schüler zu den als zu viel } \\
\text { empfundenen Hausaufgaben } \\
\text { standhaft bleiben sollen: Sie } \\
\text { hätte den Schülern entgegen- } \\
\text { kommend eine der Aufgaben } \\
\text { als Einstiegsaufgabe für die } \\
\text { kommende Stunde erlassen } \\
\text { können, aber nicht die gesam- } \\
\text { ten Hausaufgaben." }\end{array}$ & $\begin{array}{l}\text { + wird vergeben, wenn Alter- } \\
\text { nativen zu den Handlungen } \\
\text { der Lehrkraft formuliert wer- } \\
\text { den, die einen direkten und } \\
\text { konkreten Bezug zu den Or- } \\
\text { ganisationsformen, Methoden } \\
\text { und Sozialformen haben }\end{array}$ \\
\hline $\begin{array}{l}\text { Tiefen- } \\
\text { strukturen }\end{array}$ & $\begin{array}{l}\text { Formulierung von } \\
\text { Alternativen zu } \\
\text { den Handlungen } \\
\text { der/des Lehr- } \\
\text { amtsstudierenden } \\
\text { mit Bezug auf } \\
\text { die Auseinander- } \\
\text { setzung mit den } \\
\text { Lerninhalten und } \\
\text { auf die Interakti- } \\
\text { onsprozesse }\end{array}$ & $\begin{array}{l}\text { „Für zukünftige Konfronta- } \\
\text { tionen mit Schülern ist es von } \\
\text { Vorteil, dass die Lehrkraft } \\
\text { den Beschwerden dieser nicht } \\
\text { vollends nachgibt, sondern } \\
\text { auf sie eingeht. Hierbei sollte } \\
\text { sie ihnen transparent machen, } \\
\text { weshalb bspw. diese Hausauf- } \\
\text { gaben von Wichtigkeit sind, so } \\
\text { dass sie den Sinn darin sehen } \\
\text { und sie nicht als bloße Last } \\
\text { empfinden." }\end{array}$ & $\begin{array}{l}\text { + wird vergeben, wenn Alter- } \\
\text { nativen zu den Handlungen der } \\
\text { Lehrkraft formuliert werden, } \\
\text { die einen direkten und konkre- } \\
\text { ten Bezug zur Auseinanderset- } \\
\text { zung mit den Lerninhalten und } \\
\text { mit den Interaktionsprozessen } \\
\text { haben }\end{array}$ \\
\hline
\end{tabular}

In einem ersten Schritt wurden die Reflexionen der Studierenden für die Auswertung im Software-Programm MAXQDA (Kuckartz und Rädiker 2019) aufbereitet. Zur Analyse der Daten wurde die Methode der qualitativen Inhaltsanalyse gewählt (vgl. Mayring 2018; Mayring und Frenzl 2019). Als Kodiereinheit, die den minimalsten zu analysierenden Textbestandteil festlegt, wurden Hauptsätze ausgewählt. Als Kontexteinheit, die bestimmt, welche Informationen als wegweisend für die Kodierungen gelten, wurden Absätze gemäß der Reflexionsschritte Beschreiben, Begründen und Bewerten sowie Alternativen entwickeln gewählt. Nach Festlegung der Analyseeinheiten wurden die Textpassagen den entsprechenden Subkategorien sowie den dazugehörigen Unterkategorien zugeordnet. Zu Beginn der Auswertung wurden $10 \%$ der Daten durch zwei Personen unabhängig voneinander kodiert. Die Beurteilerübereinstimmung wurde mittels Cohens Kappa ermittelt und betrug $\kappa=0,65$. 
Tab. 2 Verteilung der vergebenen individuellen Codes auf den Beobachtungsgegenstand „Handeln des/der Lehramtsstudierenden“"

\begin{tabular}{lll}
\hline Gesamtzahl individuell vergebener Codes & & \\
$958(1481)$ & & \\
Positiv wahrgenommene Situation & Herausfordernd wahrgenommene Situation \\
473 (715) & $485(766)$ & \\
IG1 (alle Situationen) & IG2 (alle Situationen) & IG3 (alle Situationen) \\
258 (404) & $330(518)$ & $370(559)$ \\
\hline
\end{tabular}

Dieser Werte ist als akzeptabel zu bezeichnen (Bos 1989). Im Anschluss an die Kodierungen fand ein Austausch zur jeweils vorgenommenen Kodierung statt und die Zuordnungsregeln wurden bezüglich ihrer Eineindeutigkeit verfeinert (Mayring und Frenzl 2019). Es wurde deutlich, dass sich eine Vielzahl der Kodiereinheiten weder den Sichtstrukturen noch den Tiefenstrukturen zuordnen ließ. Das Kategoriensystem wurde daher durch die Unterkategorie Basalstrukturen ergänzt. Im Anschluss an die Pilotierungsphase erfolgte durch eine Person die Auswertung aller verbleibenden Reflexionen. Eine weitere Person kodierte unabhängig davon die Hälfte aller vorliegenden Reflexionen (Rädiker und Kuckartz 2019). Dabei wurden insgesamt 2217 individuelle Codes vergeben, 5538 inklusive der doppelt kodierten. Die Beurteilerübereinstimmung wurde anhand von 55 der insgesamt 105 Reflexionen überprüft und betrug $\kappa=0,78$. Der Wert ist als gut einzuschätzen (Bos 1989). Die insgesamt vergebenen Codes mit dem Beobachtungsgegenstand „Handeln des/der Lehramtsstudierenden" verteilen sich wie in Tab. 2 dargestellt. Ein individueller Code setzt sich dabei aus jeweils drei verschiedenen Dimensionen (Beobachtungsgegenstand; Phase im Reflexionsprozess; Reflexionsebene) zusammen.

\subsection{Auswertungsmethode: Quantitative Zusatzanalysen}

Mit einer Kontingenzanalyse und entsprechendem $\chi^{2}$-Anpassungstest wurde untersucht, ob die Häufigkeitsverteilungen der Phasen des Reflexionsprozesses (ordinalskalierte abhängige Variable) zwischen den Unterrichtssituationen (Forschungsfrage 1) sowie zwischen den einzelnen Interventionsgruppen (Forschungsfrage 2) variiert bzw. inwiefern die empirische Häufigkeitsverteilung der vergebenen Codes zwischen den Situationen bzw. Interventionsgruppen der theoretisch erwarteten Verteilung entspricht. Dabei wurden standardisierte Residuen (z-Werte) genutzt, um festzustellen inwiefern einzelne prozentuale Verteilungen häufiger als erwartet oder weniger häufig als erwartet in einzelnen Gruppen auftreten.

\section{Ergebnisse}

\subsection{Ergebnisse zur Forschungsfrage 1}

Die erste Forschungsfrage befasst sich mit der Bedeutung der reflektierten Situation für den Reflexionsprozess. Die Ergebnisse zeigen, dass im Vergleich zur positiven 
Situation (45,3\%) ein leicht geringerer Anteil der insgesamt vergebenen Codes bei der Reflexion der herausfordernden Situation auf den ersten Schritt des Beschreibens entfällt (43,8\%). Hinsichtlich des zweiten Schrittes - Begründen und Bewerten zeigen sich ebenfalls leichte Unterschiede im Hinblick auf die Art der reflektierten Situation (positiv: 37,5\%; herausfordernd: 41,9\%). Hinsichtlich des dritten Schrittes - Alternativen entwickeln - wurden bei der Reflexion der herausfordernden Situation weniger Codes vergeben $(15,1 \%)$ als bei der Reflexion der positiven Situation $(17,2 \%)$. Für den Beobachtungsgegenstand „Handeln des/der Lehramtsstudierenden“ werden insgesamt 473 Codes für positive Situationen und 485 Codes für herausfordernde Situationen vergeben. Insgesamt zeigen sich hinsichtlich aller Schritte leichte Unterschiede in den prozentualen Häufigkeiten. Statistische Zusatzanalysen zeigen jedoch keine bedeutsamen Unterschiede der prozentualen Anteile vergebener Codes in den einzelnen Phasen zwischen den Situationen, $\chi^{2}(1)=2,16, p=0,339$.

Die Auswertungen zu den Reflexionsschritten zeigen zusammenfassend, dass die Lehramtsstudierenden in ihren Reflexionen, wie theoretisch angenommen (Kleinknecht und Gröschner 2016), alle drei Schritte des Reflexionsprozesses vornehmen. Auch die komplexeren Schritte der Entwicklung von Alternativen werden sowohl bei der als positiv als auch bei der als herausfordernd wahrgenommenen Situation detailliert ausgearbeitet, wobei bei der positiven Situation geringfügig häufiger komplexe Reflexionsschritte stattfinden. Ein Beispiel für eine Reflexion, die im Schritt Alternativen entwickeln in Bezug auf eine positive Situation kodiert wurde, ist:

Als generellen Hinweis bzw. Veränderungsvorschlag schlage ich vor, dass die Schüler im Anschluss an das Unterrichtsgespräch Zeit haben, die Mindmap in ihren Unterlagen zu ergänzen, um das Erarbeitete weiter zu festigen und zu einem späteren Zeitpunkt darauf zugreifen zu können.

Ein anderer Student formuliert im Schritt Alternativen entwickeln bei der Reflexion einer herausfordernden Situation:

Für zukünftige Konfrontationen mit SuS ist es von Vorteil, dass die Lehrkraft den Beschwerden dieser nicht vollends nachgibt, sondern auf die SuS eingeht. Hierbei sollte sie den SuS transparent machen, weshalb bspw. diese Hausaufgaben von Wichtigkeit sind und die SuS den Sinn darin sehen und sie es nicht als bloße Last empfinden.

\subsection{Ergebnisse zur Forschungsfrage 2}

Die zweite Forschungsfrage befasst sich mit der Frage nach der Bedeutung des Mediums der Reflexion (eigenes vs. fremdes Video vs. Protokoll) für die Phasen des Reflexionsprozesses. Sowohl bei der Reflexion positiver als auch bei der Reflexion herausfordernder Situationen zeigt sich, dass bezüglich des ersten Schrittes - dem Beschreiben - leichte Unterschiede zwischen den Gruppen deutlich werden (positive Situation: eigenes Video: 52,2\%; Protokoll: 45,4\%; fremdes Video: 60,0\%; herausfordernde Situation: eigenes Video: 45,9\%; Protokoll: 42,8\%; fremdes Video: 48,0\%). Auch hinsichtlich des zweiten Reflexionsschrittes - dem Begründen 
und Bewerten - zeigen sich leichte Unterschiede (positive Situation: eigenes Video: 34,2\%; Protokoll: 38,6\%; fremdes Video: 31,1\%; herausfordernde Situation: eigenes Video: 38,3\%; Protokoll: 38,1\%; fremdes Video: 36,4\%). Im dritten Schritt Alternativen entwickeln - zeigen sich ebenfalls tendenziell leichte Unterschiede (positive Situation: eigenes Video: 13,7\%; Protokoll: 16,1\%; fremdes Video: 9,0\%; herausfordernde Situation: eigenes Video: 15,9\%; Protokoll: 19,1\%; fremdes Video: $15,6 \%$ ) - besonders in der Protokollbedingung scheinen situationsunabhängig komplexere Reflexionsprozesse stattzufinden. Die Unterschiede zwischen den Interventionsgruppen, die sich in den prozentualen Häufigkeiten bei den Reflexionsschritten zeigen, fallen ähnlich aus. Die statistischen Zusatzanalysen zeigen bei der Reflexion der Situationen keine statistisch bedeutsamen Unterschiede in der Komplexität der Reflexionsschritte abhängig vom Medium, $\chi^{2}(1)=8,991, p=0,061$.

\subsection{Ergebnisse zur Forschungsfrage 3}

Die dritte Forschungsfrage befasst sich mit der Bedeutung der Situation und des zugrundeliegenden Mediums für die Ebenen, wenn komplexe Schritte wie das Formulieren von Alternativen vorgenommen werden. Abb. 1 gibt Auskunft zu den prozentualen Verteilungen aller Ebenen und aller Schritte. In unserer Ergebnisdarstellung fokussieren wir uns insbesondere auf die Reflexion von Tiefenstrukturen bei komplexen Schritten, da dies entsprechend unserer Fragestellung von besonderem Forschungsinteresse für uns ist.

Hinsichtlich der Reflexion positiver Situationen wird anhand der Prozentangabe von 52,9\% in Abb. 1 deutlich, dass im Zuge des Schrittes Alternativen entwickeln, insbesondere anhand fremder Videos die Tiefenstrukturen reflektiert werden (z. B. „Ich hätte es von den SchülerInnen erkunden lassen, wie diese Aufgaben zu lösen sind, dadurch wird ihre Kreativität geweckt und sie haben beim Kontrollieren vielleicht ein höheres Verständnis als bei dieser Variante. “). Hinsichtlich der Reflexion herausfordernder Situationen zeigen die Prozentangaben von 20,5\% und 19,1\% in Abb. 1, dass während des Schrittes Alternativen entwickeln in Reflexionen insbesondere dann Tiefenstrukturen thematisiert werden, wenn anhand fremder Videos und anhand von Protokollen reflektiert wird (z. B. „Des Weiteren sollten die anderen Schülerinnen und Schüler der jeweiligen Gruppe aufgefordert werden, ihre Gruppenteilnehmer zu unterstützen bei der Ausformulierung der Argumente, statt dies als Lehrkraft selbst zu tätigen. "). Die statistischen Zusatzanalysen zeigen, dass bei der Reflexion der als positiv wahrgenommenen Situation in der Protokollbedingung häufiger als theoretisch erwartet Alternativen entwickelt werden und damit komplexe Reflexionsprozesse stattfinden, $\chi^{2}(1)=28,471, p=0,001$.

\section{Diskussion}

Das Ziel der vorliegenden Studie war es, zu untersuchen, in welchem Zusammenhang die Art der reflektierten Situation sowie das verwendete Medium mit den Reflexionsschritten stehen. Des Weiteren war es Ziel der Untersuchung, auch die Basal-, Sicht- und Tiefenstrukturen von Unterricht als Reflexionsgegenstand zu fokussieren. 


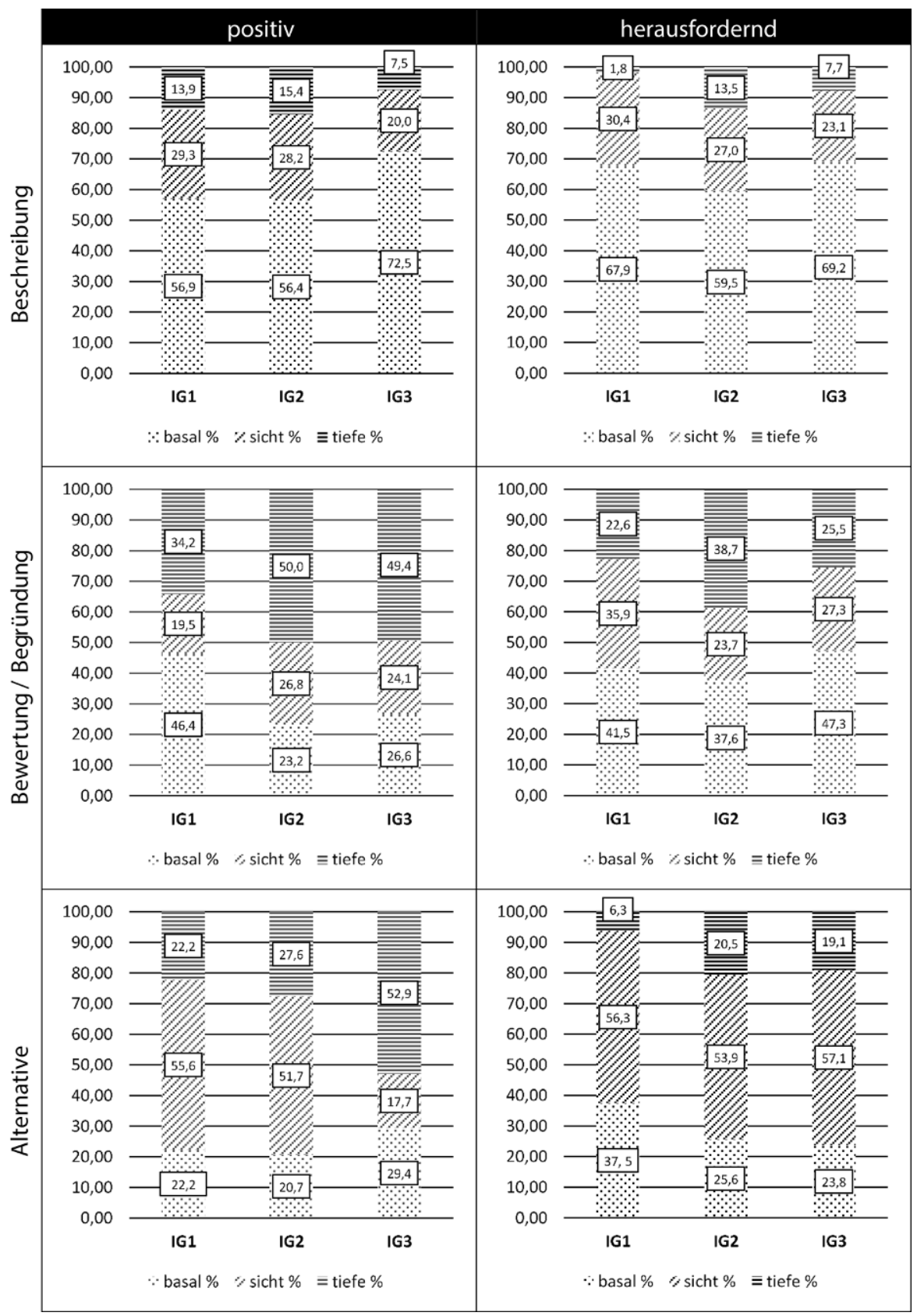

Abb. 1 Prozentuale Anteile der vergebenen Kodierungen auf den Ebenen der Reflexion verteilt nach unterschiedlichen Reflexionsschritten abhängig von der Situation und dem zugrundeliegenden Medium. ( $I G 1$ eigenes Video, IG 2 Protokoll, $I G 3$ fremdes Video) 
Zusammenfassend zeigen die Ergebnisse, dass sowohl bei der Reflexion positiver als auch bei der Reflexion herausfordernder Situationen die Tiefenstrukturen insbesondere anhand von Protokollen und anhand fremder Videos reflektiert werden. Die Ergebnisse werden nachfolgend entlang der einzelnen Forschungsfragen diskutiert.

Im Mittelpunkt der ersten Forschungsfrage steht, in welchem Zusammenhang die Art der reflektierten Situation und die drei Reflexionsschritte stehen. Unsere Annahme, dass insbesondere die Reflexion herausfordernder Situationen komplexe Reflexionsprozesse nach sich zieht (Oser und Spychinger 2005; Stark et al. 2010), konnte nicht bestätigt werden. Die Ergebnisse zeigen, dass komplexe Schritte sowohl bei der Reflexion positiver als auch bei herausfordernden Situationen vollzogen werden. Dieser Befund entspricht nicht den Ergebnissen von Heemsoth und Kleickmann (2018), in deren Studie insbesondere problematische Situationen zu komplexen Prozessen anregten. Mit Bezug auf die Befunde zur professionellen Unterrichtswahrnehmung wäre denkbar, dass es Lehramtsstudierenden schwerfällt, insbesondere herausfordernde Situationen in ihrer Komplexität wahrzunehmen (Seidel und Prenzel 2007). In der Folge könnte angenommen werden, dass ihnen auch das Begründen und Bewerten sowie das Formulieren von alternativem Unterrichtshandeln im Falle als herausfordernd wahrgenommener Situationen schwerfällt (vgl. Klassen und Durksen 2014; Schäfer und Seidel 2015). Unsere Ergebnisse verweisen darauf, dass Lehramtsstudierende bei der Reflexion herausfordernder Situationen als auch bei der Reflexion positiver Situationen dazu angeregt werden, Möglichkeiten veränderten Handelns zu entwickeln. Eine mögliche Erklärung für diesen Befund könnte sein, dass sowohl positive als auch herausfordernde Unterrichtserfahrungen von den Lehramtsstudierenden als neuartige Anforderungssituationen begriffen werden, die sie in vergleichbarer Weise dazu motivieren, sich mit den Geschehnissen im Unterricht reflexiv zu beschäftigen. Möglicherweise nehmen die Lehramtsstudierenden aufgrund fehlender Unterrichtspraxis auch gelungene Situationen als herausfordernd wahr.

Im Mittelpunkt der zweiten Forschungsfrage steht, in welchem Zusammenhang das zugrundeliegende Medium und die Komplexität der Reflexion stehen. Entsprechend der Forschungslage (Kleinknecht und Gröschner 2016) nahmen wir zum einen an, dass besonders die Reflexion anhand fremder Videos im Vergleich zu eigenen Videos zu komplexen Reflexionsschritten führt. Zum anderen nahmen wir an, dass mithilfe von Protokollen komplexe Reflexionsprozesse angeregt werden (Kramer et al. 2017; Syring et al. 2015). Es zeigt sich, dass sich unsere erste Annahme unabhängig von der Art der reflektierten Situation nicht bestätigen ließ. Lehramtsstudierende, die anhand fremder Videos reflektierten, unterschieden sich nicht von Studierenden, die anhand eigener Videos reflektierten. Unsere zweite Annahme hingegen konnte bestätigt werden, denn es zeigt sich, dass tendenziell die Reflexion anhand von Protokollen zu komplexen Reflexionsschritten anregt. Anknüpfend an empirische Befunde zu professioneller Unterrichtswahrnehmung könnte angenommen werden, dass das Nachvollziehen anhand von Videos insbesondere bei Reflexionsprozessen früher Praxiserfahrungen aufgrund der hohen Komplexität kognitiv und emotional herausfordernd ist (Reh und Schelle 2010; Syring et al. 2015). Lehramtsstudierende sind wegen fehlender Erfahrungen nur bedingt in der Lage, die Komplexität des Gesamtgeschehens zu ergründen und entsprechend damit umzugehen. Gerade 
videografierter Unterricht lässt aufgrund der Informationsdichte das komplexe Unterrichtsgeschehen deutlich werden (Krammer und Reusser 2005). In Erhebungen zeigte sich, dass dies insbesondere im Falle von Studierenden mit Beanspruchungen einhergehen kann, insbesondere dann, wenn noch nicht umfassend auf Erfahrungen im Unterrichten zurückgegriffen werden kann (vgl. Seidel et al. 2011; Kleinknecht und Gröschner 2016). Protokollbasierte Reflexionen ermöglichen es anscheinend, sich auf die zentralen Inhalte des Geschriebenen zu konzentrieren und nicht von der Fülle an Informationen, die Unterrichtsvideos liefern, abgelenkt zu werden (Kramer et al. 2017).

Im Mittelpunkt der dritten Forschungsfrage steht, anhand welchen Mediums und im Falle welcher Situation Lehramtsstudierende insbesondere die Tiefenstrukturen des Unterrichts reflektieren. Anknüpfend an die Forschung zum problembasierten Lernen wurde angenommen, dass insbesondere die Reflexion herausfordernder Situationen zur Reflexion von Tiefenstrukturen anregen (Stark et al. 2010) und dass Tiefenstrukturen sowohl anhand von Videos als auch anhand von Protokollen reflektiert werden (Kramer et al. 2017). Bestätigt werden konnte, dass insbesondere fremde Videos zur Reflexion der Tiefenstrukturen anregen, und zwar besonders dann, wenn komplexe Reflexionsschritte im Falle positiver Situationen vollzogen werden. Angenommen werden könnte, dass Studierende stark herausgefordert werden, wenn sie ihr eigenes Handeln reflektieren sollen. Denkbar wäre zudem, dass der hohe Grad an Komplexität Kapazitäten beansprucht, die Reflexionen der Tiefenstrukturen eher entgegenstehen. Reflexionen anhand fremder Videos hingegen können ermöglichen, einen Abstand zu schaffen, da nicht das eigene Handeln nachvollzogen werden muss (Häusler et al. 2019). Zudem konnte bestätigt werden, dass im Falle herausfordernder Situationen sowohl die Auseinandersetzung mit fremden Videos als auch mit Protokollen zu komplexen Reflexionen der Tiefenstrukturen anregen. Mögliche Erklärungen für unsere Befunde könnten auch sein, dass besonders jene Situationen im eigenen Unterricht als herausfordernd wahrgenommen wurden, in denen eventuell nur wenige Handlungsalternativen zur Verfügung standen. Bei der Beobachtung fremder Situationen spielt diese Ebene der spontanen Entwicklung von Handlungsalternativen vielleicht keine Rolle - so könnte es sein, dass fremde Situationen eher aufgrund der externen Umstände der Situation (z.B. Unterrichtsstörung) und nicht aufgrund des eigenen inneren Erlebens als herausfordernd bewertet wurden. Dies wäre eine mögliche Erklärung für das Ergebnis, dass fremde Videos stärker mit der Entwicklung von Alternativen assoziiert waren und damit als geeignete Übungsmöglichkeit angesehen werden können. Denkbar wäre zudem, dass durch die stattfindende Distanzierung zum Geschehen eine komplexe und umfassende Auseinandersetzung ermöglicht wird (Häusler et al. 2019). Reflexionen anhand von Protokollen könnten Lehramtsstudierenden ebenfalls ermöglichen, eine Distanzierung zum Geschehen vorzunehmen, die in irritierenden Situationen von Handlungsdruck entlastet (Kramer et al. 2017). Bestätigt werden konnte hingegen nicht vollständig, dass Tiefenstrukturen insbesondere bei herausfordernden Situationen reflektiert werden. Denkbar wäre, dass den Lehramtsstudierenden durch das Nachvollziehen positiv verlaufenden Unterrichts Erfolgserfahrungen ermöglicht werden. Dies könnte sie in ihren Selbstwirksamkeitserwartungen stärken, weiterer Alternativen zu entwickeln, die ebenfalls zu Erfolg führen (Bandura 1997). Zudem 
ist denkbar, dass insbesondere bei Reflexionen positiver Situationen anhand eigener Videos weniger Handlungsdruck wahrgenommen wird, Möglichkeiten alternativen Handelns anbringen zu müssen. Dies könnte eine Entlastung bedeuten, die Reflexionen der Tiefenstrukturen im Falle komplexer Reflexionsschritte zuträglich ist.

\subsection{Limitationen}

Limitationen der vorliegenden Studie ergeben sich hinsichtlich der Verallgemeinerbarkeit der dargestellten Befunde. Zum einen konnten bisher lediglich Lehramtsstudierende einer Universität untersucht werden. Zum anderen beziehen sich die vorliegenden Befunde lediglich auf Lehramtsstudierende im Bachelorstudiengang. Daher sind weitere Studien erforderlich, um zu überprüfen, inwieweit sich Lehramtsstudierende u.a. im Masterstudiengang unterscheiden, die aufgrund von Praktika über umfangreichere schulpraktische Erfahrungen verfügen. Zusätzlich ist zu überprüfen, inwieweit ein Prä-Post-Design entwickelt werden könnte, um Veränderungen in den Reflexionsfähigkeiten zu ergründen. Zudem ist zu überdenken, inwieweit spezifische Thematiken, beispielsweise Aspekte wie das Klassenmanagement, vorgegeben werden sollten, anstatt Lehramtsstudierende frei entscheiden zu lassen, welchen Thematiken sie sich widmen (Drechsel und Schindler 2019). Es könnte untersucht werden, inwieweit dies begünstigt, dass sich Lehramtsstudierende insbesondere den Tiefenstrukturen widmen. In der vorliegenden Studie haben wir uns gegen eine Eingrenzung der Thematik entschieden, um Lehramtsstudierende selbst entscheiden zu lassen, was sie als besonderes gelungen und als besonders herausfordernd wahrgenommen haben. Auch zukünftig ist bei der Anleitung der Lehramtsstudierenden zur Reflexion darauf zu achten, deutlich zwischen positiven - im Sinne von gut gelungenen Unterrichtssituationen - und herausfordernden - im Sinne von ungünstig verlaufenden Unterrichtssituationen - zu unterscheiden und den entsprechenden Reflexionsauftrag zu präzisieren. Weiterhin ist zu prüfen, wie Lehramtsstudierende zu ihrer individuellen Einschätzung der als positiv bzw. als herausfordernd wahrgenommenen Situationen kommen, da dies für die Bewertung der Ergebnisse bedeutend ist.

\subsection{Implikationen}

In der vorliegenden Studie konnte gezeigt werden, dass Lehramtsstudierende von angeleiteten Reflexionen hinsichtlich komplexer Reflexionsprozesse profitieren, und zwar sowohl bei der Reflexion positiver als auch herausfordernder Situationen (vgl. Heemsoth 2019). Insbesondere das differenzierte Nachvollziehen praktischer Unterrichtserfahrungen gilt als zentrales Ziel der Lehrkräftebildung, da es die Entwicklung professionsspezifischer Fähigkeiten maßgeblich unterstützen kann (vgl. Gläser-Zikuda et al. 2019). Mögliche erste Implikationen für die Lehrkräftebildung sind entsprechend unserer Studie dahingehend abzuleiten, Lehramtsstudierenden zunächst Reflexionen von als positiv wahrgenommenen Situationen zu ermöglichen. In unserer Studie zeigte sich, dass das Nachvollziehen gelungener Praxis komplexe Reflexionsprozesse begünstigt. Denkbar wäre, auf diesem Wege mit der Reflexionspraxis vertraut zu werden, so dass sich Lehramtsstudierende anschließend auch 
beim Nachvollziehen von als herausfordernd wahrgenommenen Situationen komplexen Reflexionsprozessen widmen können. Sinnvoll wäre es dabei, insbesondere Reflexionen anhand fremder Videos vornehmen zu lassen, da diese insbesondere zur Reflexion der Tiefenstrukturen anregen. Lehramtsstudierende könnten dabei mithilfe fremder Videos vor kognitiver und emotionaler Überforderung bewahrt werden. Möglicherweise könnten Lehramtsstudierende, nachdem sie den Reflexionsprozess anhand positiver Situationen verinnerlicht und die Tiefenstrukturen des Geschehens nachvollzogen haben, in der Folge hinsichtlich ihrer Reflexionsfähigkeiten von herausfordernden Situationen profitieren. In unserer Studie zeigte sich, dass komplexe Reflexionen der Sicht- und Tiefenstrukturen begünstigt werden, so dass sich sowohl Reflexionen von videografiertem als auch von protokollierten Unterrichtsmaterial empfehlen. Allerdings können an dieser Stelle nur vage Implikationen formuliert werden. Die weiterführende Untersuchung der Fragestellungen dieser Studie ist anhand umfassenderer Stichproben notwendig, um die Ergebnisse zu validieren und Implikationen ableiten zu können.

Förderung Das dieser Arbeit zugrundeliegende Vorhaben wurde im Rahmen der gemeinsamen „Qualitätsoffensive Lehrerbildung" von Bund und Ländern mit Mitteln des Bundesministeriums für Bildung und Forschung unter dem Förderkennzeichen 01JA1516 gefördert. Die Verantwortung für den Inhalt dieser Veröffentlichung liegt bei der Autorinnen.

Danksagung Wir danken herzlich unserer Mitarbeiterin Anja Henke für ihre Zuarbeit zum Manuskript und für ihre wertvollen Rückmeldungen.

Funding Open Access funding enabled and organized by Projekt DEAL.

Open Access Dieser Artikel wird unter der Creative Commons Namensnennung 4.0 International Lizenz veröffentlicht, welche die Nutzung, Vervielfältigung, Bearbeitung, Verbreitung und Wiedergabe in jeglichem Medium und Format erlaubt, sofern Sie den/die ursprünglichen Autor(en) und die Quelle ordnungsgemäß nennen, einen Link zur Creative Commons Lizenz beifügen und angeben, ob Änderungen vorgenommen wurden.

Die in diesem Artikel enthaltenen Bilder und sonstiges Drittmaterial unterliegen ebenfalls der genannten Creative Commons Lizenz, sofern sich aus der Abbildungslegende nichts anderes ergibt. Sofern das betreffende Material nicht unter der genannten Creative Commons Lizenz steht und die betreffende Handlung nicht nach gesetzlichen Vorschriften erlaubt ist, ist für die oben aufgeführten Weiterverwendungen des Materials die Einwilligung des jeweiligen Rechteinhabers einzuholen.

Weitere Details zur Lizenz entnehmen Sie bitte der Lizenzinformation auf http://creativecommons.org/ licenses/by/4.0/deed.de.

\section{Literatur}

Aebli, H. (1961). Grundformen des Lehrens. Stuttgart: Klett.

Altrichter, H. (2000). Handlung und Reflexion bei Donald Schön. In G. H. Neuweg (Hrsg.), Wissen-Können-Reflexion (S. 201-221). München: Studien-Verlag.

Bandura, A. (1997). Self-efficacy: the exercise of control. New York: W. H. Freeman.

Baumert, J., \& Kunter, M. (2006). Stichwort: Professionelle Kompetenz von Lehrkräften. Zeitschrift für Erziehungswissenschaft, 9(4), 469-520.

Berliner, D.C. (2004). Describing the behavior and documenting the accomplishments of expert teachers. Bulletin of Science, Technology \& Society, 24, 200-212.

Blömeke, S., König, J., Busse, A., Suhl, U., Benthien, J., Döhrmann, M., \& Kaiser, G. (2014). Von der Lehrerausbildung in den Beruf - Fachbezogenes Wissen als Voraussetzung für Wahrnehmung, Interpretation und Handeln im Unterricht. Zeitschrift für Erziehungswissenschaft, 17(3), 509-542. 
Bos, W. (1989). Reliabilität und Validität in der Inhaltsanalyse. Ein Beispiel zur Kategorienoptimierung in der Analyse chinesischer Textbücher für den muttersprachlichen Unterricht von Auslandschinesen. In W. Bos \& C. Tarnai (Hrsg.), Angewandte Inhaltsanalyse in Empirischer Pädagogik und Psychologie (S. 61-72). Münster: Waxmann.

Clarà, M. (2015). What is reflection? Looking for clarity in an ambiguous notion. Journal of Teacher Education, 66(3), 261-271.

Combe, A., \& Kolbe, F. U. (2008). Lehrerprofessionalität: Wissen, Können, Handeln. In W. Helsper \& J. Böhme (Hrsg.), Handbuch der Schulforschung (S. 857-875). Wiesbaden: Springer.

Dewey, J. (1933). How we think. A restatement of the relation of reflective thinking to the educative process. Boston: Heath.

Drahmann, M., Zorn, S. K., Rothland, M., \& König, J. (2018). Forschendes Lernen im Praxissemester: Das Studienprojekt als Lernprodukt. In J. König, M. Rothland \& N. Schaper (Hrsg.), Learning to practice, learning to reflect? Ergebnisse aus der Längsschnittstudie LtP zur Nutzung und Wirkung des Praxissemesters in der Lehrerbildung (S. 115-134). Wiesbaden: Springer VS.

Drechsel, B., \& Schindler, A. K. (2019). Unterrichtsqualität. In D. Urhahne, M. Dresel \& F. Fischer (Hrsg.), Psychologie für den Lehrerberuf (S. 353-372). Wiesbaden: Springer.

van Es, E.A., \& Sherin, M.G. (2002). Learning to notice: scaffolding new teachers' interpretations of classroom interactions. Society for Information Technology \& Teacher Education, 10(4), 571-596.

Gläser-Zikuda, M., Hagenauer, G., Hofmann, F., \& Wolf, N. (2019). Reflexion in Lehr-Lernprozessen. In M. Harring, C. Rohlfs \& M. Gläser-Zikuda (Hrsg.), Handbuch Schulpädagogik (S. 516-528). Münster: Waxmann.

Gröschner, A., \& Hascher, T. (2019). Praxisphasen in der Lehrerinnen- und Lehrerbildung. In M. Harring, C. Rohlfs \& M. Gläser-Zikuda (Hrsg.), Handbuch Schulpädagogik (S. 652-664). Münster: Waxmann.

Gruber, H., \& Hascher, T. (2011). Lehrer/innenexpertise zwischen Wissen und Können. In S. Rahm \& C. Nerowski (Hrsg.), Enzyklopädie Erziehungswissenschaft online (EEO), Fachgebiet Schulpädagogik. Münster: Waxmann.

Hamel, C., \& Viau-Guay, A. (2019). Using video to support teachers' reflective practice: a literature review. Cogent Education, 6, 1-14.

Hascher, T. (2014). Forschung zur Wirksamkeit der Lehrerbildung. In E. Terhart, H. Bennewitz \& M. Rothland (Hrsg.), Handbuch der Forschung zum Lehrberuf (S. 542-571). Münster: Waxmann.

Hatton, N., \& Smith, D. (1995). Reflection in teacher education: towards definition and implementation. Teaching and Teacher Education, 11(1), 33-49.

Häusler, J., Jurik, V., Schindler, A.-K., Gröschner, A., \& Seidel, T. (2019). Videografie im Unterricht. In M. Harring, C. Rohlfs \& M. Gläser-Zikuda (Hrsg.), Handbuch Schulpädagogik (S. 831-840). Münster: Waxmann.

Heemsoth, T. (2019). Unterrichtsbeispiele vergleichen. Eine experimentelle Studie mit Sportlehramtsstudierenden. German Journal of Exercise and Sport Research, 49, 45-55.

Heemsoth, T., \& Kleickmann, T. (2018). Learning to plan self-controlled physical education: good vs. problematic teaching examples. Teaching and Teacher Education, 71, 168-178.

Keller-Schneider, M. (2016). Professionalisierung ohne Beanspruchung? Diskussionsbeitrag zum Themenschwerpunkt: Burnout und Stress beim Übergang in den Lehrerberuf. Psychologie in Erziehung und Unterricht, 63, 305-314.

Klassen, R.M., \& Durksen, T.L. (2014). Weekly self-efficacy and work stress during the teaching practicum. A mixed methods study. Learning and Instruction, 33, 158-169.

Kleinknecht, M., \& Gröschner, A. (2016). Fostering preservice teachers' noticing with structured video feedback: results of an online- and video-based intervention study. Teaching and Teacher Education, 59, 45-56.

Kleinknecht, M., \& Schneider, J. (2013). What do teachers think and feel when analyzing videos of themselves and other teachers teaching? Teaching and Teacher Education, 33, 13-23.

Korthagen, F. A. J. (2010). Situated learning theory and the pedagogy of teacher education: towards an integrative view of teacher behavior and teacher learning. Teaching and Teacher Education, 26, 98-106.

Kramer, C., König, J., Kaiser, G., Ligtvoet, R., \& Blömeke, S. (2017). Der Einsatz von Unterrichtsvideos in der universitären Ausbildung: Zur Wirksamkeit video- und transkriptgestützter Seminare zur Klassenführung auf pädagogisches Wissen und situationsspezifische Fähigkeiten angehender Lehrkräfte. Zeitschrift für Erziehungswissenschaft, 20(1), 1-28.

Krammer, K., \& Reusser, K. (2005). Unterrichtsvideos als Medium der Aus- und Weiterbildung von Lehrpersonen. Beiträge zur Lehrerinnen- und Lehrerbildung, 23(1), 35-50. 
Kuckartz, U., \& Rädiker, S. (2019). Datenaufbereitung und Datenbereinigung in der qualitativen Sozialforschung. In N. Baur \& J. Blasius (Hrsg.), Handbuch Methoden der empirischen Sozialforschung (S. 441-456). Wiesbaden: Springer.

Kultusministerkonferenz (2019). Standards für die Lehrerbildung: Bildungswissenschaften. Beschluss der Kultusministerkonferenz vom 16.05.2019. Berlin: Sekretariat der Ständigen Konferenz der Kultusminister der Länder in der Bundesrepublik Deutschland.

Kunter, M., \& Ewald, S. (2016). Bedingungen und Effekte von Unterricht: Aktuelle Forschungsperspektiven aus der pädagogischen Psychologie. In N. McElvany, W. Bos, H. G. Holtappels, M. M. Gebauer \& F. Schwabe (Hrsg.), Bedingungen und Effekte guten Unterrichts (S. 9-31). Münster: Waxmann.

Landmann, M., Perels, F., Otto, B., Schnick-Vollmer, K., \& Schmitz, B. (2015). Selbstregulation und selbstgesteuertes Lernen. In E. Wild \& J. Möller (Hrsg.), Pädagogische Psychologie (S. 45-65). Berlin: Springer.

Lazarides, R. (2018). Vernetzung von Schulpraxis und Universität: Ein Seminarkonzept zur Unterrichtsentwicklung durch reflektierte Praxiserfahrungen. In A. Borowski, H. Prechtl \& A. Ehlert (Hrsg.), Ergebnisbericht zu den Aktivitäten im Rahmen der Qualitätsoffensive Lehrerbildung (2015-2018) (Potsdamer Beiträge zur Lehrerbildung und Bildungsforschung, Bd. 1, S. 189-200). Potsdam: Universitätsverlag.

Lohse-Bossenz, H., \& Krauskopf, K. (2017). Strukturiert und vernetzt - eine experimentelle Studie zu den Auswirkungen unterschiedlicher Instruktionen auf die schriftliche Reflexion von Unterrichtsvideos. 82. Jahrestagung der Arbeitsgruppe Empirisch Pädagogische Forschung, Tübingen.

Mayring, P. (2018). Qualitative Forschungsdesigns in der Psychologie. In G. Mey \& K. Mruck (Hrsg.), Handbuch Qualitative Forschung in der Psychologie (S. 1-15). Wiesbaden: Springer.

Mayring, P., \& Frenzl, T. (2019). Qualitative Inhaltsanalyse. In N. Baur \& J. Blasius (Hrsg.), Handbuch Methoden der empirischen Sozialforschung (S. 633-648). Wiesbaden: Springer.

Oser, F. K., \& Baeriswyl, F. J. (2001). Choreographies of teaching. Bridging instruction to learning. In V. Richardson (Hrsg.), Handbook of research on teaching (S. 1031-1065). Washington, DC: American Educational Research Association.

Oser, F. K., \& Spychinger, M. (2005). Lernen ist schmerzhaft. Weinheim: Beltz.

Rädiker, S., \& Kuckartz, U. (2019). Analyse qualitativer Daten mit MAXQDA. Text, Audio und Video. Wiesbaden: Springer.

Reh, S., \& Schelle, C. (2010). Der Fall im Lehrerstudium. In C. Schelle, K. Rabenstein \& S. Reh (Hrsg.), Unterricht als Interaktion. Ein Fallbuch für die Lehrerbildung (S. 13-23). Bad Heilbrunn: Klinkhardt.

Reusser, K. (2016). Jenseits der Beliebigkeit. „Konstruktivistische Didaktik“ auf dem Prüfstand der empirischen Unterrichtsforschung. Journal für LehrerInnenbildung, 2, 40-48.

Reusser, K., \& Wyss, H. (2000). Die Ausbildung der Lehrerinnen und Lehrer unterwegs zu neuen Zielen. Standortbestimmung der Schweiz. Lehrerbildung zu Beginn des neuen Jahrhunderts und Perspektiven ihrer künftigen Weiterentwicklung. Beiträge zur Lehrerinnen- und Lehrerbildung, 18(1), 7-16.

Rosaen, C. L., Lundeberg, M., Terpstra, M., Cooper, M., Fu, J., \& Niu, R. (2010). Seeing through a different lens: what do interns learn when they make video cases of their own teaching? Teacher Educator, 45(1), 1-22.

Schäfer, S., \& Seidel, T. (2015). Noticing and reasoning of teaching and learning components by preservice teachers. Journal of Educational Research Online, 7(2), 34-58.

Schneider, J., Bohl, T., Kleinknecht, M., Rehm, M., Kuntze, S., \& Syring, M. (2016). Unterricht analysieren und reflektieren mit unterschiedlichen Fallmedien: Eine Untersuchung zur vermeintlichen Überlegenheit von Video gegenüber Text. Unterrichtswissenschaft, 44(4), 474-491.

Schön, D. A. (1983). The reflective practitioner: how professionals think in action. New York: Basic Books.

Seidel, T., \& Prenzel, M. (2007). Wie Lehrpersonen Unterricht wahrnehmen und einschätzen - Erfassung pädagogisch-psychologischer Kompetenzen mit Videosequenzen. In M. Prenzel, I. Gogolin \& H.H. Krüger (Hrsg.), Kompetenzdiagnostik (S. 201-216). Wiesbaden: VS.

Seidel, T., Blomberg, G., \& Renkl, A. (2013). Instructional strategies for using video in teacher education. Teaching and Teacher Education, 34(1), 56-65.

Seidel, T., Stürmer, K., Blomberg, G., Kobarg, M., \& Schwindt, K. (2011). Teacher learning from analysis of videotaped classroom situations: Does it make a difference whether teachers observe their own teaching or that of others? Teaching and Teacher Education, 27(2), 259-267.

Stark, R., Herzmann, P., \& Krause, U.-M. (2010). Effekte integrierter Lernumgebungen - Vergleich problembasierter und instruktionsorientierter Seminarkonzeptionen in der Lehrerbildung. Zeitschrift für Pädagogik, 56(4), 548-563.

Syring, M., Bohl, T., Kleinknecht, M., Kuntze, S., Rehm, S., \& Schneider, J. (2015). Videos oder Texte in der fallbasierten Lehrerbildung? Effekte unterschiedlicher Medien auf die kognitive Belastung 
und die motivational-emotionalen Prozesse beim Lernen mit Fällen. Zeitschrift für Erziehungswissenschaft, 18(4), 667-685.

Wagner, W., Göllner, R., Werth, S., Voss, T., Schmitz, B., \& Trautwein, U. (2016). Student and teacher ratings of instructional quality: consistency of ratings over time, agreement, and predictive power. Journal of Educational Psychology, 108(5), 705-721.

Wyss, C. (2013). Unterricht und Reflexion. Eine mehrperspektivische Untersuchung der Unterrichts- und Reflexionskompetenz von Lehrkräften. Münster: Waxmann.

Wyss, C. (2018). Mündliche, kollegiale Reflexion von videografiertem Unterricht. In E. Christof, J. Köhler, K. Rosenberger \& C. Wyss (Hrsg.), Mündliche, schriftliche und theatrale Wege der Praxisreflexion. Beiträge zur Professionalisierung pädagogischen Handelns (S. 15-50). Bern: Hep. 